\title{
Infection and drug-induced toxic epidermal necrolysis: A case report.
}

\author{
Victoria Birlutiu $^{1}$, Rares Mircea Birlutiu ${ }^{2 *}$, Gabriela Mariana Iancu ${ }^{3}$ \\ ${ }^{1}$ Academic Emergency Hospital Sibiu-Infectious Diseases Clinic, Faculty of Medicine, Lucian Blaga University of \\ Sibiu, Sibiu, Romania \\ ${ }^{2}$ Faculty of Medicine Sibiu, Lucian Blaga University of Sibiu, Romania \\ ${ }^{3}$ Academic Emergency Hospital Sibiu-Dermatology Clinic, Faculty of Medicine, Lucian Blaga University of Sibiu, \\ Sibiu, Romania
}

\begin{abstract}
Lyell's syndrome or toxic epidermal necrolysis is a dermatological disease with severe evolution, and cutaneous-mucosal lesions involving more than $30 \%$ of body surface area. The following are the most commonly involved in triggering TEN: antibiotics, anti-inflammatory drugs, chemotherapeutic agents, antivirals, and anticonvulsants drugs. The evolution is accompanied by local complications (superinfections, vicious scars), or general complications (sepsis, multiple organ failure). We present the case of a patient with infectious-induced Lyell's syndrome associated with exposure to two classes of drugs at risk of inducing Lyell's syndrome (antibiotics and nonsteroidal anti-inflammatory drugs). Supportive therapy, associated with the treatment of cutaneous mucosal lesions, and the treatment of sepsis, were the most important elements that led to a favourable evolution of our case.
\end{abstract}

Keywords: Toxic epidermal necrolysis, Infection-induced, Drug-induced, TEN. Accepted on April 16, 2018

\section{Introduction}

Lyell's syndrome or toxic epidermal necrolysis (TEN) is a severe dermatological condition, in which cutaneous mucosal lesions are rapidly extending, can evolve into multiple, serious complications, sometimes even leading to death. Unlike Stevens-Johnson syndrome in which targetoid lesions do not exceed $10 \%$ of the body surface area, over $30 \%$ of the skin surface is affected in TEN. The most common etiological factors in TEN are the drugs. Other triggers recognized in TEN are infections, immunizations or immunosuppressive conditions induced by organ transplantation [1]. Up to now, more than 220 drugs have been described [2,3] as being associated with Lyell's syndrome, the most common being antibiotics (sulfonamides, macrolides, penicillins, quinolones) [1], chemotherapeutic agents, antimalarial drugs [4], antiinflammatory drugs, anticonvulsants and antiretroviral drugs.

The appearance of late-onset manifestations, 1-3 weeks after exposure to the triggering factor, is explained by the fact that in TEN, the pathogenic mechanism mimics a delayed hypersensitivity reaction at antigen primary exposure [2], but with extremely rapid and aggressive reaction at subsequent exposure. Immunopathological changes incriminated in the pathogenetic mechanism of the disease involve activation of the Fas-FasL pathway with keratinocyte apoptosis [5], activation of the perforin-granzyme pathway by cytotoxic $\mathrm{T}$ lymphocyte [6], excess release of proinflammatory cytokines
(IL, TNF, INF), and the excess synthesis of granulysin by the T lymphocyte and NK cells $[7,8]$.

This entire immunological cascade results in apoptosis and keratinocyte necrosis and secondary dermo-epidermal cleavage. At the cutaneous level, the eruption is initially localized to the cephalic extremity, but it generalizes quite quickly, consisting of targetoid erythematous-papular elements "en cocarde" with the tendency to confluence in plaques and pustules, sometimes with vesicles and bubbles on the surface. The Nicolsky's sign (dermo-epidermal cleavage upon the friction in the periphery of the lesion) is positive, expressing the fact that the disease is active, in evolution. In the mucous membranes, hyperemia, erosions and ulcerations occur, which are covered by hematic crusts, which, depending on the location, can cause dysphagia, visual disturbances, dysuria, or dyspnoea.

The therapeutic intervention in time reduces the risk of developing complications (superinfections of cutaneous mucosal lesions, retractile vicious scars, pneumonia, renal insufficiency, blindness, sepsis etc.) that may be lifethreatening. Because of the large body surface area affected (over $30 \%$ of the skin surface), TEN treatment requires surveillance in intensive care or burn wards. Particular attention is paid to skin and mucosal care by using neutral solutions for dressing and emollient creams, and antibiotics for ulcerations to prevent superinfections. In parallel, hydro- 
electrolytic and volume rebalancing is performed, as well as albumin administration, antibiotic therapy mainly antistaphylococcal and analgesic drugs administration. To reduce mortality, good interdisciplinary collaboration (dermatologistinfection diseases specialist-ophthalmologist-urologistintensive care specialist) is needed. Administration of systemic corticotherapy is questionable, and is currently a controversial topic. Other therapeutic measures that can reduce mortality in TEN include plasmapheresis, monoclonal antibody to TNFalpha, and intravenous immunoglobulins. The mortality rate varies with age, comorbidity and the extent of skin lesions (which may range from $30 \%$ to $70 \%$ ).

\section{Case Report}

We present the case of a 24-year-old Caucasian white male patient known with heart malformation (common atrioventricular canal, partial form, with total primary correction), and under antibiotic therapy (amoxicillinclavulanate) and nonsteroidal anti-inflammatory drugs (paracetamol, ibuprofen) for a dental abscess, shows flu-like symptoms (fever $39.7^{\circ} \mathrm{C}$, chills, headache, myalgia, photophobia) on the seventh day of treatment, morbiliform erythematous-papular rash, initially in the cephalic extremity, with rapid thoraco-abdominal and limb extension. The patient quickly associates mucosal lesions, respectively oculopalpebral ulcers, ulcerations of lips, mouth and genital mucosa. The patient presents itself to the Emergency Room and is admitted in the Dermatology Clinic.

Upon admission: feverish patient $\left(38.3^{\circ} \mathrm{C}\right)$, with altered general condition, intense headache, photophobia, erythematous-papular-vesicular disseminated eruption, more pronounced in the cephalic extremity with lesions arranged "en cocarde", confluated in plaques and papules with irregular contours, well defined, with spontaneous cleavage on mild friction (Nicolsky's positive sign), pressure-induced cleavage on the bubble that caused the lesion to spread (Asboe-Hansen's positive sign).

At the level of the semimucosa of the lips and the oral mucosa, there were extensive ulcerations covered by hematic crusts and fibrinous-purulent fetid secretions, being impossible to feed himself. At ocular level, one can see conjunctival hyperaemia, purulent conjunctival secretions, palpebral edema with narrowing of the palpebral opening. At the level of the left toe, periungually, he shows erythema and painful edema, secondary to the slight enclavation of the nail at this level (Figure 1).

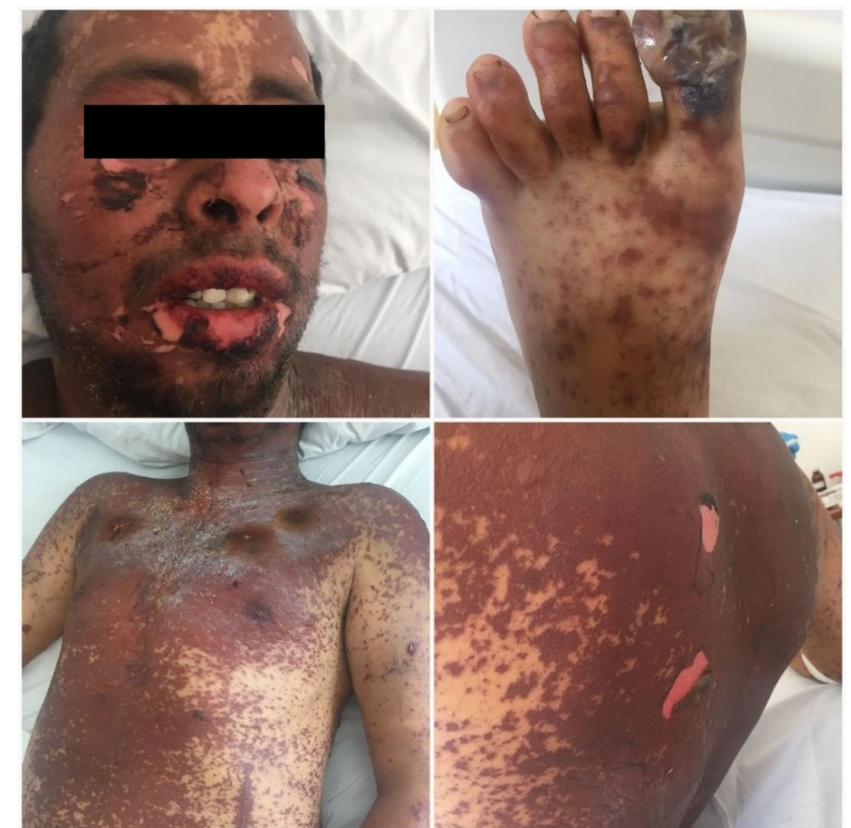

Figure 1. Clinical aspect, 2 days after admission: generalized hyperchromic erythematous plaques affecting about 55\% of the skin surface, with important cutaneous-mucosal decollation; punctionated bubble in the left toe.

Associated, he presented submandibular adenopathies of about $2 \mathrm{~cm}$, painful on examination. The patient was tachycardic, a heart rate of 120 beats per minute with a systolic murmur in the mitral and aortic focal area, a blood presaure of $90 / 50 \mathrm{mmHg}$, hepatomegaly of $1.5 \mathrm{~cm}$, and without signs of meningeal irritation. Initially, laboratory investigations revealed mild leukocytosis, thrombocytopenia, inflammatory syndrome, and mild hyperglycemia. Serology for HIV, syphilis, hepatitis B and $\mathrm{C}$, and haemocultures were negative. Bacteriological examinations from the left toe periungual secretion were positive for Staphylococcus aureus, CA-MRSA (sensitive to Gentamicin, Ciprofloxacin, Rifampin, Chloramphenicol, Linezolid, Cotrimoxazole) and E .coli sensitive to Aztreonam, Imipenem, Meropenem, Ceftazidime, Cefotaxime. The laboratory examinations are presented in Table 1. The completion of investigations with procalcitonin dosage confirmed the evolution to TEN complications with sepsis, the patient being transferred into the Infectious Diseases Clinic.

Table 1. Evolution of the main laboratory examinations during hospitalization.

\begin{tabular}{llll}
\hline Laboratory examination & Day $\mathbf{1}$ & Day $\mathbf{3}$ & Day $\mathbf{1 0}$ \\
\hline Procalcitonin & $14 \mathrm{ng} / \mathrm{ml}$ & $0.16 \mathrm{ng} / \mathrm{ml}$ & negative \\
\hline C reactive protein & $19.10 \mathrm{mg} / \mathrm{L}$ & $9 \mathrm{mg} / \mathrm{L}$ & $5 \mathrm{mg} / \mathrm{L}$ \\
\hline Le $\mu$ cocytes & $10.690 \times 10^{3} / \mu \mathrm{l}$ & $0-5 \mathrm{mg} / \mathrm{L}$ & $10120 \times 10^{3} / \mu \mathrm{l}$ \\
\hline Ne $\mu$ trophils & $9.01 \times 10^{3}$ & $4.76 \times 10^{3} / \mu \mathrm{l}$ & $4-10 \times 10^{3} / \mu \mathrm{l}$ \\
\hline
\end{tabular}




\begin{tabular}{|c|c|c|c|c|}
\hline Thrombocytes & $122 \times 10^{3} / \mu \mathrm{l}$ & $122 \times 10^{3} / \mu \mathrm{l}$ & $497 \times 10^{3} / \mu \mathrm{l}$ & $150-400 \times 10^{3} / \mu \mathrm{l}$ \\
\hline Erythrocyte sedimentation rate & $34 \mathrm{~mm} / \mathrm{h}$ & $23 \mathrm{~mm} / \mathrm{h}$ & NA & $0-15 \mathrm{~mm} / \mathrm{h}$ \\
\hline Fibrinogen & $492.8 \mathrm{mg} / \mathrm{dl}$ & $472.2 \mathrm{mg} / \mathrm{dl}$ & NA & $180-380 \mathrm{mg} / \mathrm{dl}$ \\
\hline Glycaemia & $135 \mathrm{mg} / \mathrm{dL}$ & $134 \mathrm{mg} / \mathrm{dL}$ & $127 \mathrm{mg} / \mathrm{dL}$ & $70-105 \mathrm{mg} / \mathrm{dL}$ \\
\hline Haemocultures & negative & negative & NA & \\
\hline Blood $\mu$ rea nitrogen & $43 \mathrm{mg} / \mathrm{dL}$ & $36 \mathrm{mg} / \mathrm{dL}$ & $44 \mathrm{mg} / \mathrm{dL}$ & $19-44 \mathrm{mg} / \mathrm{dL}$ \\
\hline Creatinine & $1.12 \mathrm{mg} / \mathrm{dl}$ & $0.97 \mathrm{mg} / \mathrm{dl}$ & $0.82 \mathrm{mg} / \mathrm{dl}$ & $0.7-1.3 \mathrm{mg} / \mathrm{dl}$ \\
\hline
\end{tabular}

*NA: Not Available

The examination in the ear, nose and throat sphere showed moderate edema of lutea, free vocal cords without laryngeal edema. The ophthalmic examination revealed central corneal erosion of the left eye. Systemic treatment included hydroelectrolytic rebalancing, pulse corticotherapy $(500 \mathrm{mg}$ Metilprednisolone/day) for 3 days, proton pump inhibitors, antibiotherapy (Clindamycin $1800 \mathrm{mg} /$ day with Gentamicin $240 \mathrm{mg} /$ day, then Meropenem 4g/day plus Linezolid $2 \times 600$ $\mathrm{mg} /$ day), and antifungal therapy (Fluconazole $150 \mathrm{mg} /$ day). Cutaneous mucosal ulceration was washed with saline and Kanamycin ointment was applied to mucosal ulcers and Dermazin to cutaneous lesions. The morbiliform lesions were treated with medium-potency dermatocorticoids (hydrocortisone butyrate). At ocular level, physiological saline dressing was performed, and corneal epithelization ointment (Corneogen) was applied. After obtaining the control of the sepsis, the patient was transferred to the burn ward for the reepithelialization of the extensive palmar plantar ulceration. The topical therapies with epithelization ointments and the removal of the palmar plantar affected skin allowed for rapid, healing of the remaining ulcerations without sequela (Figure 2).

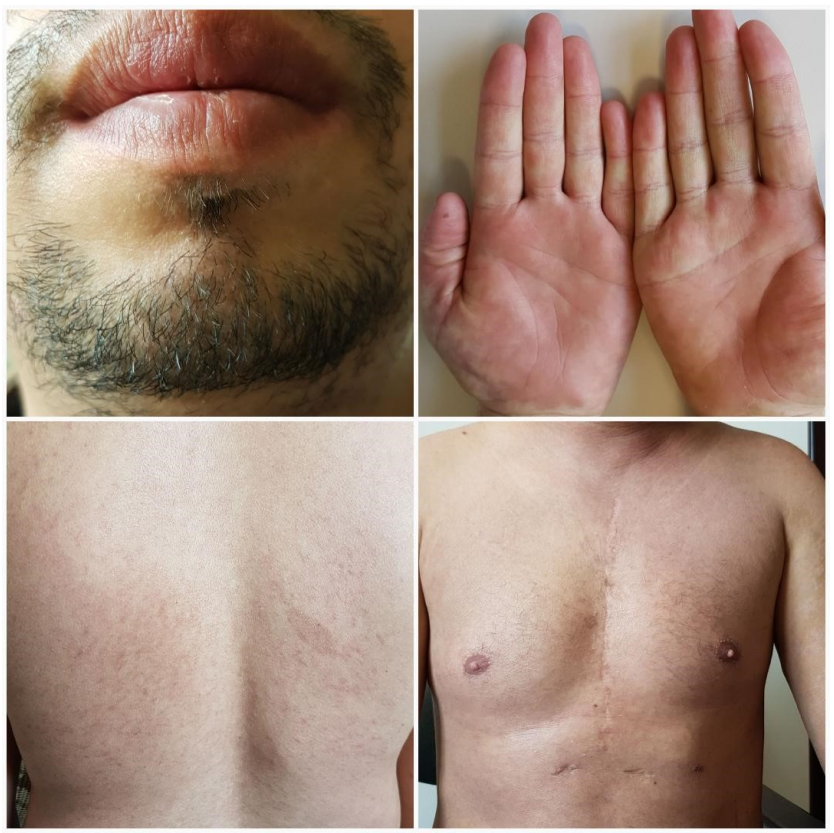

Figure 2. Clinical appearance 1 month after discharge: discrete residual hyperchromic macules and mild palmar erythema.

\section{Discussions}

TEN is a dermatological emergency, with post-drug etiology in $80-95 \%$ of cases [9]. The occurrence of drug hypersensitivity is greater in certain circumstances: the association of antibiotics in patients with cystic fibrosis, of cotrimoxazole in patients with HIV infection receiving abacavir treatment. Genetic determinism cases are described, such as in the Asian population, HLA-B $* 1502(+)$ genes, posing a risk of severe allergic reactions to carbamazepim while HLA-B $* 5801(+)$ increases TEN risk to allopurinol administration $[10,11]$.

Table 2. SCORTEN value calculation.

\begin{tabular}{ll}
\hline Predictive factors & Value \\
\hline Age over 40 & 0 \\
\hline Tachycardia (AV over $120 \mathrm{bpm})$ & 1 \\
\hline Personal history of neoplasia & 0 \\
\hline More than 10\% BSA in the first day & 1 \\
\hline Urea more than $28 \mathrm{mg} / \mathrm{dl}$ & 1 \\
\hline Glycaemia over $252 \mathrm{mg} / \mathrm{dl}$ & 0 \\
\hline Bicarbonate below $20 \mathrm{mEq} / \mathrm{l}$ & 0 \\
\hline Total & 3 \\
\hline
\end{tabular}

*BSA: Body Surface Area

The occurrence of TEN is associated in most cases with the administration of antibiotics (most commonly being sulfonamides), non-steroidal or steroidal anti-inflammatory drugs, methotrexate, allopurinol or antiretroviral medication, anticonvulsants, and anxiolytics. The case presented was in treatment with an antibiotic and anti-inflammatory combination, along with the evolution of an infection in the dental sphere but also with periungual nail infection, from which Staphylococcus aureus and E. coli were isolated. Most likely, the trigger was mixed: the periungual infection evolving towards sepsis, under antibiotic treatment inappropriate for the identified etiologies and drug-induced (amoxiline-clavulanate, ibuprofen).

Being a life-threatening disease with high mortality risk $(30-70 \%)$ [12], it is essential that the disease is properly 
managed. The use of severity of the illness score for the toxic epidermal of necrolysis (SCORTEN) is important in assessing the severity of the disease. In our case, the items in the SCORTEN calculation (Table 2) showed a value of 3 , indicating a $35 \%$ mortality risk.

High dose methylprednisolone has been shown to be effective, even if the benefits are confirmed in some retrospective studies [13], and refuted in others [14]. In parallel, we combined the antibiotic therapy appropriate to isolated bacteria, avoiding the medication at risk of cross-reactions of hypersensitivity. Intravenous immunoglobulins, plasmapheresis or biological agents (infliximab), cyclophosphamide, cyclosporine, pentoxifylline or $\mathrm{N}$-acetylcysteine, considered as third-line agents in the treatment of TEN [15], were not required.

\section{Conclusion}

Supportive therapy, associated with the treatment of cutaneous mucosal lesions, the treatment of sepsis, were the most important elements that led to healing. The particularity of the case lies in the appearance of the TEN, the triggers being polymicrobial sepsis and medication.

\section{Ethics approval}

Written informed consent was obtained from the patient for publication of this case report and any accompanying images. The study was accepted by the Ethics Committee of the hospital and they encouraged publishing the article.

\section{References}

1. https://emedicine.medscape.com/article/229698-overview

2. Chave TA, Mortimer NJ, Sladden MJ, Hall AP, Hutchinson PE. Toxic epidermal necrolysis: Current evidence, practical management and future directions. $\mathrm{Br}$ J Dermatol 2005; 153: 241-253.

3. Lissia M, Mulas P, Bulla A, Rubino C. Toxic epidermal necrolysis (Lyell's disease) Burns 2010; 36: 152-163.

4. Nunes JM, Santareno S, Guerreiro L, Margalho AF. Lyell's Syndrome and Antimalarials: A Case Report and Clinical Review. J Glob Infect Dis 2017; 9: 23-30.

5. Abe R, Shimizu T, Shibaki A, Nakamura H, Watanabe H, Shimizu H. Toxic epidermal necrolysis and StevensJohnson syndrome are induced by soluble Fas ligand. Am J Pathol 2003; 162: 1515-1520.

6. Posadas SJ, Padial A, Torres MJ, Mayorga C, Leyva L, Sanchez E. Delayed reactions to drugs show levels of perforin, granzyme B, and Fas-L to be related to disease severity. J Allergy Clin Immunol 2002; 109: 155-161.
7. Nassif A, Moslehi H, Le Gouvello S, Bagot M, Lyonnet L, Michel L. Evaluation of the potential role of cytokines in toxic epidermal necrolysis. J Invest Dermatol 2004; 123: 850-855.

8. Chung WH, Hung SI, Yang JY, Su SC, Huang SP, Wei CY. Granulysin is a key mediator for disseminated keratinocyte death in Stevens-Johnson syndrome and toxic epidermal necrolysis. Nat Med 2008; 14: 1343-1350.

9. Schwartz RA, McDonough PH, Lee BW. Toxic epidermal necrolysis: Part I. Introduction, history, classification, clinical features, systemic manifestations, etiology, and immunopathogenesis. J Am Acad Dermatol 2013; 69: 173.

10. Pirmohamed M. Genetics and the potential for predictive tests in adverse drug reactions. Chem Immunol Allergy 2012; 97: 18-31.

11. Kaniwa N, Saito Y, Aihara M, Matsunaga K, Tohkin M, Kurose K. HLA-B locus in Japanese patients with antiepileptics and allopurinol-related Stevens-Johnson syndrome and toxic epidermal necrolysis. Pharmacogenomics 2008; 9: 1617-1622.

12. Schwartz RA, McDonough PH, Lee BW. Toxic epidermal necrolysis: Part II. Prognosis, sequelae, diagnosis, differential diagnosis, prevention, and treatment. J Am Acad Dermatol 2013; 69: 187.

13. Kardaun SH, Jonkman MF. Dexamethasone pulse therapy for Stevens-Johnson syndrome/toxic epidermal necrolysis. Acta Derm Venereol 2007; 87: 144-148.

14. Schneck J, Fagot JP, Sekula P, Sassolas B, Roujeau JC, Mockenhaupt M. Effects of treatments on the mortality of Stevens-Johnson syndrome and toxic epidermal necrolysis: A retrospective study on patients included in the prospective EuroSCAR study. J Am Acad Dermatol 2008; 58: 33-40.

15. Tiwari P, Panik R, Bhattacharya A, Ahirwar D, Chandy A. Toxic epidermal necrolysis: an update. Asian Pac J Trop Dis 2013; 3: 85-92.

\section{*Correspondence to}

Rares Mircea Birlutiu

Faculty of Medicine

Lucian Blaga University of Sibiu

Romania 